\title{
Tracing evolution reveals new biomarkers
}

Recognizing
cancer as an
evolutionary
disease
may help
to improve
cancer
diagnostics

Intratumour heterogeneity (ITH) often confounds cancer biomarkers involving tumour material obtained using traditional methods. Furthermore, biopsy sampling is invasive and not feasible in all patients. Now, data from the TRACERx non-small-cell lung cancer (NSCLC) consortium demonstrate how these challenges might be addressed using RNA sequencing (RNA-seq), the T cell receptor (TCR) repertoire and/or circulating tumour cells (CTCs).

Dhruva Biswas, first author on the RNA-seq study, explains: "We set out to design a clonal expression biomarker, using our understanding of cancer evolution to look for genes that are consistently expressed across tumours and not affected by where you place the biopsy needle." To address this challenge (which can lead to discordance rates of $50 \%$ using published prognostic signatures), gene expression was analysed in tissue samples from 156 tumour regions from 48 patients, and each gene was assigned a heterogeneity score. Genes with the lowest level of ITH, but the maximum level of intertumour heterogeneity, were identified and selectively included in a validation set of 242 genes, which outperformed genes with higher levels of ITH in an independent validation cohort. From these candidate genes, an elastic-net regression machine-learning algorithm was used to generate a more practical 23-gene signature termed the outcome risk-associated clonal (ORACLE) biomarker.

ORACLE resulted in reduced levels of discordant classification

Credit: Simon Bradbrook/Springer Nature Limited

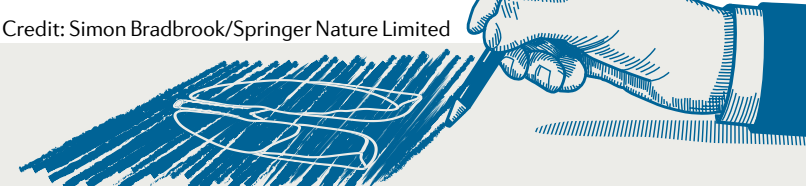

relative to other signatures in the TRACERx NSCLC cohort (11\% versus 29-43\%). Furthermore, validation of ORACLE revealed a robust association with overall survival independent of other clinical variables, indicating that ORACLE provides additive prognostic value.

Senior author Nicolai Birkbak adds: "We ran a preliminary pan-cancer analysis, in which we found that our lung-specific clonally expressed genes may hold prognostic power across multiple histologies."

Despite these promising findings, RNA-seq alone cannot entirely account for ITH. Thus, Kroopa Joshi and colleagues investigated the TCR repertoires of 72 patients from the TRACERx NSCLC cohort with early stage, treatment-naive NSCLC.

A robust positive correlation was revealed between intratumoural TCR diversity and genomic diversity. Furthermore, TCRs were either present in all samples (ubiquitous) or in a subset of samples (regional) and were correlated with the presence of clonal and subclonal alterations, and more-diverse and less-diverse 'clusters' of TCR CDR3 regions, respectively. This implies that TCR diversity reflects the spatial distribution of tumourassociated antigens. Intriguingly, intratumoural TCRs were also detected in patients' blood samples, indicating potential clinical utility. Senior author Benny Chain summarizes: "We were able to detect these same T cells in the blood at the time of surgical tumour removal, thus raising the possibility of a blood test that can reliably tell us how well a treatment is working for that individual patient."

CTCs have the potential to provide an additional level of prognostic information, although detecting these cells in patients with early stage NSCLC has previously proved challenging. In TRACERx, CellSearch-detectable CTCs were present in pulmonary venous blood samples (PV-CTCs) obtained at surgical resection in 48 of 100 patients. Having $>7$ PV-CTCs per $7.5 \mathrm{ml}$ blood sample was strongly associated with shorter time to disease relapse, and patients with higher numbers of PV-CTCs had worse outcomes. However, not all of these PV-CTCs were tumour derived: several had a genomic profile suggesting a nonmalignant epithelial origin.

Mutations in individual CTCs from one patient were found to be highly concordant with those obtained from a subsequent metastatic lesion (91\%), relative to the primary tumour (71\%), despite being sampled 10 months before the emergence of metastatic disease.

First author Francesca Chemi summarizes "CTCs found in the tumour-draining pulmonary vein of patients with early stage NSCLC can provide an 'early warning sign' for eventual metastatic relapse." Senior author Ged Brady comments on future directions of this research: "We aim to further evaluate the prognostic potential of the number of epithelial cells and establish if this could translate into improved post-operative surveillance; we also aim to establish if the phylogenetic relationships between PV-CTCs, the primary tumour and the metastatic lesion we observed in our first patient are indeed the norm."

Consortium lead Charles Swanton concludes: "Recognizing cancer as an evolutionary disease may help to improve cancer diagnostics. We are actively seeking to validate these findings in larger cohorts, with the aim of reducing cancer-associated death."

Peter Sidaway

ORIGINAL ARTICLES Biswas, D. et al. Nat. Med. 25 , 1540-1548 (2019) | Joshi, K. et al. Nat. Med. 25, 1549-1559 (2019) |Chemi, F. et al. Nat. Med. 25 , 1534-1539 (2019) 